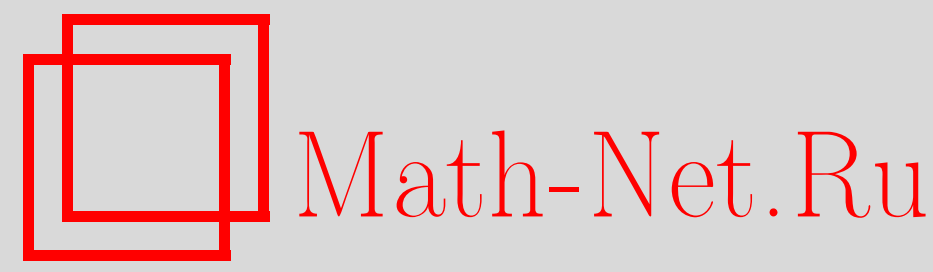

M. А. Скопина, Ортогональные полиномиальные базисы Шаудера в $C[-1,1]$ с оптимальным ростом степеней, Матем. сб., 2001, том 192, номер 3, 115-136

DOI: https://doi.org/10.4213/sm553

Использование Общероссийского математического портала Math-Net.Ru подразумевает, что вы прочитали и согласны с пользовательским соглашением http://www . mathnet.ru/rus/agreement

Параметры загрузки:

IP: 54.164 .48 .24

26 апреля 2023 г., 14:08:49 


\title{
М. А. Скопина \\ Ортогональные полиномиальные базисы Шаудера в $C[-1,1]$ с оптимальным ростом степеней
}

\author{
Для любого $\varepsilon>0$ строится ортогональньй базис Шаудера в пространстве \\ $C[-1,1]$, состоящий из алгебраических многочленов $P_{n}$ степени не вьше $n(1+\varepsilon)$. \\ Рост степеней является минимально возможньм. \\ Библиографоия: 13 названий.
}

\section{§1. Введение}

В 1961 году П. Л. Ульянов [1] поставил задачу: каким можно сделать минимальньй рост степеней многочленов, образующих базис (или ортогональный базис) Шаудера в пространстве $C[a, b]$ ? Исследования многих авторов посвящены этой и близким к ней проблемам. Подробное изложение истории вопроса приведено, например, в [2], мы отметим лишш ее ключевые моменты. В 1914 году Г. Фабер [3] доказал, что не существует последовательности алгебраических многочленов $P_{n}$ степени $n$, образующих базис Шаудера в $C[a, b]$. В [4] Ал. А. Привалов усилил этот результат, показав, что если $P_{n}$ образуют базис Шаудера в $C[a, b]$, то существует такое $\varepsilon>0$, что степень $P_{n}$ не меньше $n(1+\varepsilon)$ для $n \geqslant n_{0}$. А в [5] он установил окончательность этой оценки, построив пример полиномиального базиса Шаудеpa, степени которого растут не быстрее, чем $n(1+\varepsilon)$. Однако этот базис не был ортогональньм. Параллельно рассматривалась аналогичная задача для тригонометрических полиномов. Ее удалось решить благодаря использованию всплесков. Д. Оффин, К. Осколков [6] заметили, что незначительная модификация всплесков Мейера дает ортогональный базис Шаудера, в котором степень полиномов лишш в два раза превышает минимальную. Заменив обычное всплеск-разложение на пакет всплесков, Р. А. Лоренц, А.А. Саакян [7] окончательно решили задачу для тригонометрических полиномов. Перенести этот результат на отрезок с помощью стандартного метода индуцированных функций не удается. В работе Т. Килгоре, Я. Престина, К. Селиж [8] задача решена для алгебраических многочленов, ортогональных с весом Чебышёва первого рода. Для многочленов, ортогональных без веса, до сих пор вопрос оставался открытым. Ответу на него посвящена настояшая работа. Идея построения базиса заимствована из [7]. Реализацию этой идеи осложняли следующие обстоятельства. Определение базисов всплесков (пакетов всплесков) связано с конструкцией кратномасштабного анализа, состояшего из пространств, инвариантных относительно сдвига. При обычном понимании оператора сдвига его нельзя рассматривать на пространствах функций, заданных на

Работа вьполнена при поддержке Российского фонда фундаментальных исследований (грант № 00-01-00467).

$$
\text { (C) М.А. СкопинА } 2001
$$


отрезке, так как сдвиг выводит за пределы пространства. Мы вводим обобщенные операторы сдвига так, что сохраняются все необходимые свойства сдвига, в частности сдвиг алгебраического многочлена есть многочлен той же степени. Кроме того, мы используем подход к периодическому кратномасштабному анализу, данньй автором в [9], вместо стандартного (периодизации кратномасштабного анализа в $\left.L_{2}(\mathbb{R})\right)$. При таком подходе понятия кратномасштабного анализа и всплесков естественным образом распространяются на произвольное гильбертово пространство.

Сформулируем основной результат.

ТЕОРема 1. Для любого $\varepsilon_{0}>0$ существует последовательность алгебраических многочленов $T_{n}$ степени не выше $n\left(1+\varepsilon_{0}\right)$, попарно ортогональных на отрезке $[-1,1]$ и образующих базис Шаудера в пространстве $C[-1,1]$.

\section{§2. Кратномасштабный анализ в гильбертовом пространстве}

Пусть $H$ - гильбертово пространство со скалярньм произведением $\langle f, g\rangle, f, g \in$ $H,\left\{X_{n}\right\}_{n=-\infty}^{\infty}-$ ортонормированный базис в $H$. Если $f \in H$, то $f=\sum_{k} \hat{f}(k) X_{k}$, где $\widehat{f}(k)=\left\langle f, X_{k}\right\rangle$ суть коэффициенты Фурье элемента $f$. Для каждого неотрицательного целого $j$ определим оператор $S_{j}$ :

$$
S_{j} f=\sum_{k \in \mathbb{Z}} e^{2 \pi i 2^{-j} k} \widehat{f}(k) X_{k}
$$

Ясно, что $S_{j}$ - мультипликаторы, действующие из $H$ в $H$, и $\left\|S_{j} f\right\|=\|f\|$ для любого $f \in H$. В случае, когда $H=L_{2}(-\pi, \pi), X_{n}(t)=e^{i n t}, S_{j}$ являются операторами сдвига (на $\left.2^{-j+1} \pi\right)$. И в общем случае их также естественно называть операторами сдвига, поскольку сохраняется основное свойство: последовательное применение $S_{j_{1}}, S_{j_{2}}$ дает “сдвиг” на $2 \pi\left(2^{-j_{1}}+2^{-j_{2}}\right)$.

Tеорема 2. Пусть $G_{j} \in H, V_{j}=\operatorname{span}\left\{S_{j}^{n} G_{j}, n=0, \ldots, 2^{j}-1\right\}, j \in \mathbb{Z}_{+}$. Если коэффициенты Фурье $G_{j}$ удовлетворяют условиям:

G1. $\widehat{G}_{0}(k)=0$ для любого $k \neq 0$;

G2. для любого $j \in \mathbb{Z}_{+}$и любого $k=0, \ldots, 2^{j}-1$ существует такое $m \in \mathbb{Z}$, чmo $\widehat{G}_{j}\left(2^{j} m+k\right) \neq 0$;

G3. для любого $k \in \mathbb{Z}$ существует такое $j \in \mathbb{Z}_{+}$, ито $\widehat{G}_{j}(k) \neq 0$;

G4. для любого $j=1,2, \ldots$ и любого $k \in \mathbb{Z}$ существует такое комплексное число $\delta_{k}^{j}$, что $\widehat{G}_{j-1}\left(2^{j} m+k\right)=\delta_{k}^{j} \widehat{G}_{j}\left(2^{j} m+k\right)$ для всех $m \in \mathbb{Z}$,

mo

1. $\left\{S_{j}^{n} G_{j}\right\}_{n=0}^{2^{j}-1}-$ базис в $V_{j}$;

1а. $\left\{S_{j}^{n} G_{j}\right\}_{n=0}^{2^{j}-1}$ является ортонормированным базисом тогда и только тогда, когда

$$
\sum_{m \in \mathbb{Z}}\left|\widehat{G}_{j}\left(2^{j} m+k\right)\right|^{2}=2^{-j}
$$

для всех $k \in \mathbb{Z}$;

2. $V_{j} \subset V_{j+1}$ для любого $j \in \mathbb{Z}_{+}$;

3. $\bigcup_{j=0}^{\infty} V_{j}$ плотно в $H$. 
Семейство подпространств $V_{j}$, удовлетворяющих условиям $1,2,3$ теоремы 2 , назовем кратномасштабным анализом в $H$, а последовательность элементов $G_{j}-$ масштабирующей последовательностью.

Для каждого $j \in \mathbb{Z}_{+}$и $n=0, \ldots, 2^{j}-1$ определим оператор $\omega_{n}^{j}$ :

$$
\omega_{n}^{j} f=\sum_{m \in \mathbb{Z}} \widehat{f}\left(2^{j} m+n\right) X_{2^{j} m+n}
$$

Ясно, что

$$
S_{j}^{n} f=\sum_{k=0}^{2^{j}-1} e^{2 \pi i 2^{-j} n k} \omega_{k}^{j} f
$$

Поскольку $\left\langle\omega_{k}^{j} f, \omega_{\ell}^{j} f\right\rangle=0$ при $k \neq \ell$, из этого равенства и унитарности матрицы $\left\{2^{-j / 2} e^{2 \pi i 2^{-j} n k}\right\}_{k, n=0}^{2^{j}-1}$ вытекает следующее утверждение.

Лемма 3. Пусть $f \in H$, тогда для любого $j=0,1, \ldots$

1. $\operatorname{span}\left\{S_{j}^{n} f, n=0, \ldots, 2^{j}-1\right\}=\operatorname{span}\left\{\omega_{n}^{j} f, n=0, \ldots, 2^{j}-1\right\}$;

2. система $\left\{S_{j}^{n} f\right\}_{n=0}^{2^{j}-1}$ ортогональна тогда и только тогда, когда $\left\|\omega_{n}^{j} f\right\|$ не зависит от $n$, при $\left\|\omega_{n}^{j} f\right\|=2^{-j / 2}$ она является ортонормированной.

ДоКАЗАТЕЛЬСТво ТЕОРЕмЫ 2. Утверждения 1 , 1а следуют из леммы 3. Кроме того, по лемме $3\left\{\omega_{n}^{j} G_{j}\right\}_{n=0}^{2^{j}-1}-$ базис в $V_{j}$. Поэтому утверждение 2 следует из равенства

$$
\begin{aligned}
\omega_{k}^{j} G_{j}= & \sum_{\ell \in \mathbb{Z}} \widehat{G}_{j}\left(2^{j+1} \ell+k\right) X_{2^{j+1} \ell+k}+\sum_{\ell \in \mathbb{Z}} \widehat{G}_{j}\left(2^{j+1} \ell+2^{j}+k\right) X_{2^{j+1} \ell+2^{j}+k} \\
= & \delta_{k}^{j+1} \sum_{\ell \in \mathbb{Z}} \widehat{G}_{j+1}\left(2^{j+1} \ell+k\right) X_{2^{j+1} \ell+k} \\
& \quad+\delta_{2^{j}+k}^{j+1} \sum_{\ell \in \mathbb{Z}} \widehat{G}_{j+1}\left(2^{j+1} \ell+2^{j}+k\right) X_{2^{j+1} \ell+2^{j}+k} \\
& =\delta_{k}^{j+1} \omega_{k}^{j+1} G_{j+1}+\delta_{2^{j}+k}^{j+1} \omega_{2^{j}+k}^{j+1} G_{j+1} .
\end{aligned}
$$

Для доказательства утверждения 3 достаточно проверить, что $X_{r} \in \overline{\bigcup_{j=0}^{\infty} V_{j}}$ для всех $r \in \mathbb{Z}$. Положим

$$
v_{n}^{0}=G_{0}, \quad v_{n}^{j}=\left\{\begin{array}{ll}
\omega_{n}^{j} G_{j}, & \text { если } \delta_{n}^{j}=0, \\
\omega_{n}^{j} v_{n}^{j-1}, & \text { если } \delta_{n}^{j} \neq 0,
\end{array} \quad j=1,2, \ldots\right.
$$

В силу свойства G3 существует такое $j_{0}$, что $\widehat{G}_{j_{0}}(r) \neq 0$. Отсюда и из $\mathrm{G} 4$ следует, что $\delta_{r}^{j} \neq 0$ при $j \geqslant j_{0}$. Поэтому $\widehat{v}_{r}^{j}(r)=\widehat{v}_{r}^{j_{0}}(r) \neq 0$ для всех $j \geqslant j_{0}$. Можно считать $r<2^{j_{0}}-1$. Тогда для $j \geqslant j_{0}$

$$
s_{2^{j-1}}\left(\widehat{v}_{r}^{j}(r) X_{r}-v_{r}^{j}\right)=0
$$


где $s_{N}(f)-N$-я частичная сумма Фурье элемента $f$. Покажем, что при $j \geqslant j_{0}$ справедливо равенство

$$
\widehat{v}_{r}^{j}(r) X_{r}-v_{r}^{j}=2^{j_{0}-j} \sum_{n=0}^{2^{j-j_{0}}-1} e^{-2 \pi i 2^{-j} n r} S_{j}^{n}\left(\widehat{v}_{r}^{j_{0}}(r) X_{r}-v_{r}^{j_{0}}\right)
$$

Ясно, что

$$
\widehat{v}_{r}^{j}(r) X_{r}=\widehat{v}_{r}^{j_{0}}(r) X_{r}=2^{j_{0}-j} \sum_{n=0}^{2^{j-j_{0}}-1} e^{-2 \pi i 2^{-j} n r} S_{j}^{n}\left(\widehat{v}_{r}^{j_{0}}(r) X_{r}\right)
$$

Учитывая соотношение

$$
\sum_{n=0}^{N-1} e^{2 \pi i N^{-1} n k}= \begin{cases}2^{N}, & \text { если } k=N \ell, \ell \in \mathbb{Z}, \\ 0, & \text { если } k \neq N \ell, \ell \in \mathbb{Z},\end{cases}
$$

имеем

$$
\begin{aligned}
& \sum_{n=0}^{2^{j-j_{0}}-1} e^{-2 \pi i 2^{-j} n r} S_{j}^{n} v_{r}^{j_{0}} \\
& =\sum_{n=0}^{2^{j-j_{0}}-1} e^{-2 \pi i 2^{-j} n r} \sum_{k \in \mathbb{Z}} e^{2 \pi i 2^{-j} n\left(2^{j_{0}} k+r\right)} \widehat{v}_{r}^{j_{0}}\left(2^{j_{0}} k+r\right) X_{2^{j_{0}} k+r} \\
& =\sum_{k \in \mathbb{Z}} \widehat{v}_{r}^{j_{0}}\left(2^{j_{0}} k+r\right) X_{2^{j_{0}} k+r} \sum_{n=0}^{2^{j-j_{0}}-1} e^{2 \pi i 2^{j_{0}-j} n k} \\
& =2^{j-j_{0}} \sum_{\ell \in \mathbb{Z}} \widehat{v}_{r}^{j_{0}}\left(2^{j} \ell+r\right) X_{2^{j}} \ell+r \\
&
\end{aligned}
$$

Сопоставляя соотношения (2), (3), получаем

$$
\begin{aligned}
& \left\|\widehat{v}_{r}^{j}(r) X_{r}-v_{r}^{j}\right\|=\| 2^{j_{0}-j} \sum_{n=0}^{2^{j-j_{0}-1}} e^{-2 \pi i 2^{-j} n r} S_{j}^{n}\left(\widehat{v}_{r}^{j_{0}}(r) X_{r}-v_{r}^{j_{0}}\right) \\
& -s_{2^{j-1}}\left(2^{j_{0}-j} \sum_{n=0}^{2^{j-j_{0}}-1} e^{-2 \pi i 2^{-j} n r} S_{j}^{n}\left(\widehat{v}_{r}^{j_{0}}(r) X_{r}-v_{r}^{j_{0}}\right)\right) \| \\
& =\left\|2^{j_{0}-j} \sum_{n=0}^{2^{j-j_{0}}-1} e^{-2 \pi i 2^{-j} n r} S_{j}^{n}\left(v_{r}^{j_{0}}(r) X_{r}-v_{r}^{j_{0}}-s_{2^{j-1}}\left(\widehat{v}_{r}^{j_{0}}(r) X_{r}-v_{r}^{j_{0}}\right)\right)\right\| \\
& \leqslant 2^{j_{0}-j} \sum_{n=0}^{2^{j-j_{0}}-1}\left\|\widehat{v}_{r}^{j_{0}}(r) X_{r}-v_{r}^{j_{0}}-s_{2^{j-1}}\left(\widehat{v}_{r}^{j_{0}}(r) X_{r}-v_{r}^{j_{0}}\right)\right\| \\
& =\left\|\widehat{v}_{r}^{j_{0}}(r) X_{r}-v_{r}^{j_{0}}-s_{2^{j-1}}\left(\widehat{v}_{r}^{j_{0}}(r) X_{r}-v_{r}^{j_{0}}\right)\right\| .
\end{aligned}
$$


Осталось заметить, что правая часть этого соотношения стремится к нулю при $j \rightarrow \infty$.

Для каждого $j \in \mathbb{Z}_{+}$определим элемент $H_{j}$, задав его коэффициенты Фурье:

$$
\widehat{H}_{j}(r)=e^{\pi i 2^{-j}} r{\overline{\delta_{2^{j}+r}^{j+1}}}_{G_{j+1}}(r)
$$

и положим

$$
W_{j}=\operatorname{span}\left\{S_{j}^{n} H_{j}, n=0, \ldots, 2^{j}-1\right\} .
$$

Назовем $W_{j}$ пространством всплесков.

ТЕОРема 4. Если в условиях теоремы 2 вылолнено (1), то

1. $\left\{S_{j}^{n} H_{j}\right\}_{n=0}^{2^{j}-1}$ - ортонормированныи базис в $W_{j}$,

2. $W_{j} \oplus V_{j}=V_{j+1}$.

ДокАЗАТЕЛЬСТВО. По определению функций $H_{j}$

$$
\left\langle S_{j}^{n} H_{j}, S_{j}^{m} H_{j}\right\rangle=\sum_{r \in \mathbb{Z}}\left|\widehat{G}_{j+1}(r)\right|^{2}\left|\delta_{2^{j}+r}^{j+1}\right|^{2} e^{2 \pi i 2^{-j}(n-m) r}
$$

Отсюда, учитьвая $2^{j+1}$-периодичность последовательности $\delta_{k}^{j+1}$ и условие (1), получаем

$$
\begin{aligned}
\left\langle S_{j}^{n} H_{j}, S_{j}^{m} H_{j}\right\rangle & =\sum_{k=0}^{2^{j+1}-1}\left|\delta_{2^{j}+k}^{j+1}\right|^{2} e^{2 \pi i 2^{-j}(n-m) k} \sum_{\ell \in \mathbb{Z}}\left|\widehat{G}_{j+1}\left(2^{j+1} \ell+k\right)\right|^{2} \\
& =2^{-j-1} \sum_{k=0}^{2^{j}-1}\left(\left|\delta_{2^{j}+k}^{j+1}\right|^{2}+\left|\delta_{k}^{j+1}\right|^{2}\right) e^{2 \pi i 2^{-j}(n-m) k}
\end{aligned}
$$

С другой стороны, из свойств G4 и (1) следует

$$
\begin{aligned}
\left\langle S_{j}^{n} G_{j}, S_{j}^{m} G_{j}\right\rangle= & \sum_{r \in \mathbb{Z}}\left|\widehat{G}_{j}(r)\right|^{2} e^{2 \pi i 2^{-j}(n-m) r} \\
= & \sum_{k=0}^{2^{j+1}-1}\left(\sum_{\ell \in \mathbb{Z}}\left|\delta_{k}^{j+1} \widehat{G}_{j+1}\left(2^{j+1} \ell+k\right)\right|^{2}\right. \\
& \left.\quad+\sum_{\ell \in \mathbb{Z}}\left|\delta_{2^{j}+k}^{j+1} \widehat{G}_{j+1}\left(2^{j+1} \ell+2^{j}+k\right)\right|^{2}\right) e^{2 \pi i 2^{-j}(n-m) k} \\
& =2^{-j-1} \sum_{k=0}^{2^{j}-1}\left(\left|\delta_{2^{j}+k}^{j+1}\right|^{2}+\left|\delta_{k}^{j+1}\right|^{2}\right) e^{2 \pi i 2^{-j}(n-m) k}
\end{aligned}
$$

Отсюда и из ортонормированности системы $\left\{S_{j}^{n} G_{j}\right\}_{n=0}^{2^{j}-1}$ следует утверждение 1 теоремы 4. 
Ввиду $2^{j+1}$-периодичности последовательности $\delta_{k}^{j+1}$ имеем

$$
\begin{aligned}
& \omega_{k}^{j} H_{j}=\omega_{k}^{j+1} H_{j}+\omega_{k+2^{j}}^{j+1} H_{j} \\
& =e^{\pi i 2^{-j}} \bar{k} \overline{\delta_{2^{j}+k}^{j+1}} \omega_{k}^{j+1} G_{j+1}+e^{\pi i 2^{-j}\left(k+2^{j}\right)} \overline{\delta_{2^{j+1} k}^{j+1}} \omega_{2^{j+k}}^{j+1} G_{j+1} \\
& \left.=e^{\pi i 2^{-j} k} \overline{\left(\bar{\delta}_{2^{j}+k}^{j+1}\right.} \omega_{k}^{j+1} G_{j+1}-\overline{\delta_{k}^{j+1}} \omega_{2^{j+k}}^{j+1} G_{j+1}\right) \text {. }
\end{aligned}
$$

Отсюда и из леммы 3 следует $W_{j} \subset V_{j+1}$. Кроме того, по свойству G4

$$
\omega_{k}^{j} G_{j}=\delta_{k}^{j+1} \omega_{k}^{j+1} G_{j+1}+\delta_{2^{j}+k}^{j+1} \omega_{2^{j}+k}^{j+1} G_{j+1}
$$

Это равенство и (5) составляют линейную систему относительно неизвестных $\omega_{k}^{j+1} G_{j+1}, \omega_{2^{j+k}}^{j+1} G_{j+1}$. Определитель системы равен $e^{\pi i 2^{-j} k}\left(\left|\delta_{k}^{j+1}\right|^{2}+\left|\delta_{2^{j}+k}^{j+1}\right|^{2}\right)$. По определению чисел $\delta_{k}^{j}$ (см. G4) в силу свойства G2 по крайней мере одно из чисел $\delta_{k}^{j+1}, \delta_{2^{j}+k}^{j+1}$ отлично от нуля. Следовательно, определитель не равен нулю. Отсюда и из леммы 3, принимая во внимание, что $\operatorname{dim} V_{j}+\operatorname{dim} W_{j}=\operatorname{dim} V_{j+1}$, получаем утверждение 2 теоремы 4 .

\section{§3. Построение базиса}

Пусть $H=L_{2}[-1,1], P_{n}, n=0,1, \ldots,-$ ортонормированные многочлены Лежандра. Положим $X_{0}=P_{0}, X_{n}=\frac{1}{\sqrt{2}}\left(P_{2 n}+i P_{2 n-1}\right), X_{-n}=\bar{X}_{n}$ для $n=1,2, \ldots$ Ясно, что $\left\{X_{n}\right\}_{n \in \mathbb{Z}}-$ ортонормированный базис в $H$. Будем использовать символ $\operatorname{deg} T$ для обозначения степени алгебраического многочлена $T$, рассматриваемого как полином по системе $X_{n}$, т.е. $\operatorname{deg} T=n$ для многочленов степени $2 n$ и $2 n-1$.

Рассмотрим функцию $\varphi$ с преобразованием Фурье $\widetilde{\varphi}$, обладающим свойствами: $\operatorname{supp} \tilde{\varphi} \subset\left[-\frac{1}{2}(1+\varepsilon), \frac{1}{2}(1+\varepsilon)\right], \widetilde{\varphi} \equiv 1$ на $\left[-\frac{1}{2}(1-\varepsilon), \frac{1}{2}(1-\varepsilon)\right], 0<\varepsilon \leqslant \frac{1}{6}, \widetilde{\varphi}^{2}(x)+$ $\widetilde{\varphi}^{2}(x+1)=1$ для всех $x \in \mathbb{R}, \widetilde{\varphi} \in C^{\infty}(\mathbb{R})$. Такая функция $\varphi$ является масштабируюшей (см., например, [10; гл. 5]) для обобшенных всплесков Мейера, рассмотренных в [6]. Масштабируюшее уравнение для $\varphi$ имеет вид: $\widetilde{\varphi}(2 x)=m_{0}(x) \widetilde{\varphi}(x)$, где $m_{0}-1$-периодическая функция, $m_{0}(x)=\widetilde{\varphi}(2 x)$ на $[-1 / 2,1 / 2]$. Положим $G_{j}=\sum_{k \in \mathbb{Z}} \widehat{G}_{j}(k) X_{k}$, где $\widehat{G}_{j}(k)=2^{-j / 2} \widetilde{\varphi}\left(2^{-j} k\right)$. Ввиду финитности $\widetilde{\varphi}$ функции $G_{j}$ суть полиномы, $\operatorname{deg} G_{j} \leqslant 2^{j-1}(1+\varepsilon)$. Нетрудно проверить, что последовательность $G_{j}$ является масштабируюшей, т.е. вьполнены условия G1-G4 теоремы 2 при $\delta_{r}^{j}=2^{1 / 2} m_{0}\left(2^{-j} r\right)$. Таким образом, построен кратномасштабньй анализ в $L_{2}[-1,1]$ и определены соответствующие ему последовательность всплесков $H_{j}$ и пространства всплесков $W_{j}$. Поскольку условие $(1)$, очевидно, выполнено, по теоремам 2,4 базисы сдвигов $\left\{S_{j}^{n} G_{j}\right\}_{n=0}^{2^{j}-1},\left\{S_{j}^{n} H_{j}\right\}_{n=0}^{2^{j}-1}$ являются ортонормированными. Введем в рассмотрение еще последовательность $\lambda_{r}^{j}=$ $2^{1 / 2} e^{2 \pi i 2^{-j}} m_{0}\left(2^{-j} r+\frac{1}{2}\right)$ и отметим следующие очевидные свойства чисел $\delta_{r}^{j}, \lambda_{r}^{j}$ :

$$
\begin{aligned}
\left|\delta_{r}^{j}\right|^{2}+\left|\lambda_{r}^{j}\right|^{2}= & \left|\delta_{r}^{j}\right|^{2}+\left|\delta_{r+2^{j-1}}^{j}\right|^{2}=\left|\lambda_{r}^{j}\right|^{2}+\left|\lambda_{r+2^{j-1}}^{j}\right|^{2}=2 \\
& \delta_{r}^{j} \overline{\lambda_{r}^{j}}+\delta_{r+2^{j-1}}^{j} \overline{\lambda_{r+2^{j-1}}^{j}}=0
\end{aligned}
$$


Пусть $r \in \mathbb{Z}, k \in \mathbb{Z}_{+}$, обозначим через $\Delta_{k}$ и $\Lambda_{k}$ мультипликаторы, заданные на $H$ и определяемые равенствами

$$
\widehat{\Delta_{k} f}(r)=\delta_{r}^{k} \widehat{f}(r), \quad \widehat{\Lambda_{k} f}(r)=\lambda_{r}^{k} \widehat{f}(r) .
$$

Зафиксируем натуральное $s$. Для целого $j \geqslant s$ и $\nu=\left(\nu_{j-s+1}, \ldots, \nu_{j}\right)$, где $\nu_{k}$ принимает значения 0 и 1 , положим

$H_{j}^{\nu}=H_{j}^{s, \nu}=\prod_{k=j-s+1}^{j} \Delta_{k}^{\nu_{k}} \Lambda_{k}^{1-\nu_{k}} H_{j}, \quad W_{j}^{\nu}=\operatorname{span}\left\{S_{j-s}^{n} H_{j}^{\nu}, n=0, \ldots, 2^{j-s}-1\right\}$.

Поскольку обе последовательности $\left\{\delta_{r}^{j}\right\},\left\{\lambda_{r}^{j}\right\} 2^{j}$-периодичны, то

$$
\begin{aligned}
\omega_{r}^{j} H_{j}^{\nu} & =\sum_{\ell \in \mathbb{Z}} \prod_{k=j-s+1}^{j}\left(\delta_{2^{j} \ell+r}^{k}\right)^{\nu_{k}}\left(\lambda_{2^{j} \ell+r}^{k}\right)^{1-\nu_{k}} \widehat{H}_{j}\left(2^{j} \ell+r\right) X_{2^{j} \ell+r} \\
& =\prod_{k=j-s+1}^{j}\left(\delta_{r}^{k}\right)^{\nu_{k}}\left(\lambda_{r}^{k}\right)^{1-\nu_{k}} \omega_{r}^{j} H_{j} .
\end{aligned}
$$

Отсюда и из леммы 3 , принимая во внимание, что $\omega_{r}^{j-s} H_{j}^{\nu}=\sum_{\ell=0}^{2^{s}-1} \omega_{r+2^{j-s}}^{j} H_{j}^{\nu}$, получаем $W_{j}^{\nu} \subset W_{j}$. Теперь покажем, что при $\nu \neq \nu^{\prime} W_{j}^{\nu} \perp W_{j}^{\nu^{\prime}}$ и при каждом $\nu$ система функций $\left\{S_{j-s}^{n} H_{j}^{\nu}\right\}_{n=0}^{2^{j-s}-1}$ является ортонормированным базисом пространства $W_{j}^{\nu}$. В силу леммы 3 для этого достаточно установить, что

$$
\left\langle\omega_{r}^{j-s} H_{j}^{\nu}, \omega_{r}^{j-s} H_{j}^{\nu^{\prime}}\right\rangle=0
$$

при $\nu \neq \nu^{\prime}$ и

$$
\left\|\omega_{r}^{j-s} H_{j}^{\nu}\right\|^{2}=2^{-j+s} .
$$

Для доказательства этих равенств проведем индукцию по $s$. Пусть сначала $s=1$. Применяя (8), имеем

$$
\begin{aligned}
& \left\langle\omega_{r}^{j-1} H_{j}^{\nu}, \omega_{r}^{j-1} H_{j}^{\nu^{\prime}}\right\rangle=\left\langle\omega_{r}^{j} H_{j}^{\nu}, \omega_{r}^{j} H_{j}^{\nu^{\prime}}\right\rangle+\left\langle\omega_{r+2^{j-1}}^{j} H_{j}^{\nu}, \omega_{r+2^{j-1}}^{j} H_{j}^{\nu^{\prime}}\right\rangle \\
& =\left(\delta_{r}^{j}\right)^{\nu_{j}}\left(\overline{\delta_{r}^{j}}\right)^{\nu_{j}^{\prime}}\left(\lambda_{r}^{j}\right)^{1-\nu_{j}}\left(\overline{\lambda_{r}^{j}}\right)^{1-\nu_{j}^{\prime}}\left\|\omega_{r}^{j} H_{j}\right\|^{2} \\
& \quad+\left(\delta_{r+2^{j-1}}^{j}\right)^{\nu_{j}}\left(\overline{\bar{\delta}_{r+2^{j-1}}^{j}}\right)^{\nu_{j}^{\prime}}\left(\lambda_{r+2^{j-1}}^{j}\right)^{1-\nu_{j}}\left(\overline{\lambda_{r+2^{j-1}}^{j}}\right)^{1-\nu_{j}^{\prime}}\left\|\omega_{r+2^{j-1}}^{j} H_{j}\right\|^{2} .
\end{aligned}
$$

Отсюда, учитывая, что $\left\|\omega_{k}^{j} H_{j}\right\|^{2}=2^{-j}$, и применяя равенства $(6),(7)$, получаем $(9),(10)$ для $s=1$. Теперь предположим, что равенства $(9),(10)$ верны для всех $s<s_{0}$. Пусть $\nu=\left(\nu_{j-s_{0}+1}, \ldots, \nu_{j}\right), \nu^{\prime}=\left(\nu_{j-s_{0}+1}^{\prime}, \ldots, \nu_{j}^{\prime}\right)$, положим $\mu=\left(\nu_{j-s_{0}+2}, \ldots, \nu_{j}\right), \mu^{\prime}=\left(\nu_{j-s_{0}+2}^{\prime}, \ldots, \nu_{j}^{\prime}\right), \varkappa=\nu_{j-s_{0}+1}, \varkappa^{\prime}=\nu_{j-s_{0}+1}^{\prime}$. Тогда

$$
\begin{aligned}
& \left\langle\omega_{r}^{j-s_{0}} H_{j}^{\nu}, \omega_{r}^{j-s_{0}} H_{j}^{\nu^{\prime}}\right\rangle=\left\langle\omega_{r}^{j-s_{0}+1} H_{j}^{\nu}, \omega_{r}^{j-s_{0}+1} H_{j}^{\nu^{\prime}}\right\rangle+\left\langle\omega_{r+2^{j-s_{0}}}^{j-s_{0}+1} H_{j}^{\nu}, \omega_{r+2^{j-s_{0}}}^{j-s_{0}+1} H_{j}^{\nu^{\prime}}\right\rangle \\
& \left.\left.=\left(\delta_{r}^{j-s_{0}+1}\right)^{\varkappa} \overline{\left(\delta_{r}^{j-s_{0}+1}\right.}\right)^{\varkappa^{\prime}}\left(\lambda_{r}^{j-s_{0}+1}\right)^{1-\varkappa} \overline{\left(\lambda_{r}^{j-s_{0}+1}\right.}\right)^{1-\varkappa^{\prime}}\left\langle\omega_{r}^{j-s_{0}+1} H_{j}^{\mu}, \omega_{r}^{j-s_{0}+1} H_{j}^{\mu^{\prime}}\right\rangle \\
& \left.\left.+\left(\delta_{r+2^{j-s_{0}}}^{j-s_{0}+1}\right)^{\varkappa} \overline{\left(\delta_{r+2^{j-s_{0}}}^{j-s_{0}+1}\right.}\right)^{\varkappa^{\prime}}\left(\lambda_{r+2^{j-s_{0}}}^{j-s_{0}+1}\right)^{1-\varkappa} \overline{\left(\lambda_{r+2^{j-s_{0}}}^{j-s_{0}+1}\right.}\right)^{1-\varkappa^{\prime}}\left\langle\omega_{r+2^{j-s_{0}}}^{j-s_{0}+1} H_{j}^{\mu}, \omega_{r+2^{j-s_{0}}}^{j-s_{0}+1} H_{j}^{\mu^{\prime}}\right\rangle .
\end{aligned}
$$


По индукционному предположению $\left\langle\omega_{k}^{j-s_{0}+1} H_{j}^{\mu}, \omega_{k}^{j-s_{0}+1} H_{j}^{\mu^{\prime}}\right\rangle=0$ при $\mu \neq \mu^{\prime}$ и $\left\langle\omega_{k}^{j-s_{0}+1} H_{j}^{\mu}, \omega_{k}^{j-s_{0}+1} H_{j}^{\mu^{\prime}}\right\rangle=2^{j-s_{0}+1}$ при $\mu=\mu^{\prime}$. Отсюда, применяя равенства $(6),(7)$, получаем $(9),(10)$ для $s=s_{0}$.

Таким образом, доказано, что

$$
W_{j}=\bigoplus_{\nu} W_{j}^{\nu}
$$

и система функций $\left\{S_{j-s}^{n} H_{j}^{\nu}\right\}_{n, \nu}$ является ортонормированным базисом пространства $W_{j}$. Эта система называется пакетом всплесков.

Пусть $T$ - алгебраический многочлен, $\operatorname{deg} T \leqslant m 2^{k-1}(1+\varepsilon), \varepsilon \leqslant 1 /(4 m)$. Поскольку на множестве $\left\{\left[\ell+\frac{1}{2}-\frac{1}{4}(1-\varepsilon), \ell+\frac{1}{2}+\frac{1}{4}(1-\varepsilon)\right], \ell \in \mathbb{Z}\right\}$ функция $m_{0}$ тождественно равна нулю, $\operatorname{deg} \Delta_{k} T \leqslant\left(m 2^{k-1}-2^{k-2}\right)(1+\varepsilon)$ при нечетных $m$ и $\operatorname{deg} \Lambda_{k} T \leqslant\left(m 2^{k-1}-2^{k-2}\right)(1+\varepsilon)$ при четных $m$. Отсюда, принимая во внимание, что $\operatorname{deg} H_{j} \leqslant 2^{j}(1+\varepsilon)$, и считая $\varepsilon \leqslant 2^{-s-2}$, нетрудно понять, что функции $H_{j}^{s, \nu}$ (для фиксированных $j, s$ ) можно перенумеровать одномерньм индексом $\ell=1, \ldots, 2^{s}$ таким образом, чтобы

$$
\operatorname{deg} H_{j}^{s, \ell} \leqslant\left(2^{j-1}+\ell 2^{j-s-1}\right)(1+\varepsilon),
$$

положив $\ell=2^{s}-\left(\alpha_{1} 2^{s-1}+\alpha_{2} 2^{s-2}+\cdots+\alpha_{s}\right)$, где

$$
\begin{aligned}
& \alpha_{1}=\left\{\begin{array}{l}
0, \text { если } \nu_{j}=1, \\
1, \text { если } \nu_{j}=0,
\end{array}\right. \\
& \alpha_{k+1}=\left\{\begin{array}{l}
0, \text { если } \alpha_{k}=1, \nu_{j-k}=0 \text { или } \alpha_{k}=0, \nu_{j-k}=1, \\
1, \text { если } \alpha_{k}=0, \nu_{j-k}=0 \text { или } \alpha_{k}=1, \nu_{j-k}=1 .
\end{array}\right.
\end{aligned}
$$

Положим

$T_{1}=G_{0}$

$T_{2^{j}+n+1}=H_{j}^{j, n+1} \quad$ для $j<s, n=0, \ldots, 2^{j}-1$,

$T_{2^{j}+m 2^{j-s}+r+1}=S_{j-s}^{r} H_{j}^{s, m+1}$ для $j \geqslant s, m=0, \ldots, 2^{s}-1, r=0, \ldots, 2^{j-s}-1$.

Неравенство (12), очевидно, влечет $\operatorname{deg} T_{N} \leqslant \frac{1}{2} N(1+\varepsilon)$ при $N \leqslant 2^{s}$. Представляя произвольное натуральное $N>2^{s}$ в виде $N=2^{j}+m 2^{j-s}+r+1$, где $j \geqslant s$, $m<2^{s}, r<2^{j-s}$, из (12) получаем

$$
\begin{aligned}
\operatorname{deg} T_{N} & \leqslant\left(2^{j-1}+(m+1) 2^{j-s-1}\right)(1+\varepsilon) \\
& =\frac{1}{2}\left(2^{j}+m 2^{j-s}+2^{j-s}\right)(1+\varepsilon) \leqslant \frac{1}{2} N\left(1+2^{-s}\right)(1+\varepsilon) .
\end{aligned}
$$

По любому заданному $\varepsilon_{0}>0$ можно подобрать столь большое натуральное $s$ и столь малое $\varepsilon>0$, чтобы для всех $N$ выполнялось неравенство

$$
\operatorname{deg} T_{N} \leqslant \frac{1}{2} N\left(1+\varepsilon_{0}\right) .
$$


Далее нам будут полезны следующие представления функций $H_{j}, H_{j}^{s, \nu}(\nu-$ бинарньй вектор):

$$
H_{j}=2^{-j / 2} \sum_{\ell \in \mathbb{Z}} \widetilde{\psi}\left(2^{-j} \ell\right) X_{\ell}, \quad H_{j}^{s, \nu}=2^{-j / 2} \sum_{\ell \in \mathbb{Z}} \widetilde{\psi}^{s, \nu}\left(2^{-j} \ell\right) X_{\ell},
$$

где $\widetilde{\psi}, \widetilde{\psi}^{s, \nu}$ - функции, определяемые равенствами

$$
\begin{aligned}
\widetilde{\psi}(x) & =e^{\pi i x} m_{0}\left(\frac{x+1}{2}\right) \widetilde{\varphi}\left(\frac{x}{2}\right) \\
\widetilde{\psi}^{s, \nu} & =\prod_{k=j-s+1}^{j}\left(m_{0}(x)\right)^{\nu_{k}}\left(e^{2 \pi i x} m_{0}\left(x+\frac{1}{2}\right)\right)^{1-\nu_{k}} \widetilde{\psi}(x) .
\end{aligned}
$$

В соответствии с описанной вьше перенумерацией функций $H_{j}^{s, \nu}$ в функциях $\psi^{s, \nu}$ мы также заменим $\nu$ на одномерный индекс $\ell=1, \ldots, 2^{s}$.

\section{§4. Вспомогательные результаты}

Нам понадобятся следующие свойства ортонормированных многочленов Лежандра $P_{n}$. Их можно найти, например, в монографии [11].

Формула Дирихле-Мелера:

$$
P_{n}(\cos \pi \theta)=\sqrt{2\left(n+\frac{1}{2}\right)} \int_{0}^{\theta} \frac{\cos \left(\left(n+\frac{1}{2}\right) \pi \tau\right) d \tau}{\sqrt{\cos \pi \tau-\cos \pi \theta}}, \quad \theta \in(0,1) .
$$

Уравнение Вольтерра второго рода:

$$
\begin{aligned}
& (\sin \pi \theta)^{1 / 2} P_{n}(\cos \pi \theta)=\lambda_{n} \cos \left(\left(n+\frac{1}{2}\right) \pi \theta-\frac{\pi}{4}\right) \\
& \quad-\frac{\pi}{\left(n+\frac{1}{2}\right)} \int_{\theta}^{1 / 2} \frac{\sin \left(\left(n+\frac{1}{2}\right) \pi(t-\theta)\right)}{4 \sin ^{2} \pi t}(\sin \pi t)^{1 / 2} P_{n}(\cos \pi t) d t
\end{aligned}
$$

где $\theta \in(0,1)$

$$
\lambda_{n}=\sqrt{\frac{2}{\pi}}+O\left(\frac{1}{n^{2}}\right) .
$$

При тех же значениях $\theta$ и $\lambda_{n}$ справедлива асимптотическая формула

$$
(\sin \pi \theta)^{1 / 2} P_{n}(\cos \pi \theta)=\lambda_{n} \cos \left(\left(n+\frac{1}{2}\right) \pi \theta-\frac{\pi}{4}\right)+O\left(\frac{1}{n \sin \pi \theta}\right) .
$$

Метод Лиувилля-Стеклова (см. [11; 8.61$])$ дает следующее уточнение этой формулы

$$
\begin{aligned}
& (\sin \pi \theta)^{1 / 2} P_{n}(\cos \pi \theta)=\lambda_{n} \cos \left(\left(n+\frac{1}{2}\right) \pi \theta-\frac{\pi}{4}\right) \\
& \quad+\frac{\lambda_{n}}{8\left(n+\frac{1}{2}\right)} \sin \left(\left(n+\frac{1}{2}\right) \pi \theta-\frac{\pi}{4}\right) \operatorname{ctg} \pi \theta+O\left(\frac{1}{n^{2} \sin ^{2} \pi \theta}\right) .
\end{aligned}
$$


Для всех $x \in[-1,1]$ имеет место неравенство

$$
\left|P_{n}(x)\right| \leqslant P_{n}(1)=\sqrt{n+\frac{1}{2}} .
$$

Будем обозначать через $C$ абсолютные постоянные, вообще говоря, различные.

Лемма 5. Пусть $\theta \in(0,1), n \in \mathbb{Z}, n \neq 0$, тогда $X_{n}(\cos \pi \theta)$ есть сумма двух слагаемых вида

$$
\frac{1}{\sqrt{\sin \pi \theta}}\left[A_{1}(\theta) \xi_{1}(n) e^{2 \pi i n \theta}+A_{2}(\theta) \xi_{2}(n) e^{-2 \pi i n \theta}\right]+O\left(\frac{1}{|n|(\sin \pi \theta)^{3 / 2}}\right)
$$

или

$$
\begin{aligned}
& \frac{1}{\sqrt{\sin \pi \theta}}\left[A_{1}(\theta) \xi_{1}(n) e^{2 \pi i n \theta}+A_{2}(\theta) \xi_{2}(n) e^{-2 \pi i n \theta}\right] \\
& \quad+\frac{1}{n(\sin \pi \theta)^{3 / 2}}\left[A_{3}(\theta) \xi_{3}(n) e^{2 \pi i n \theta}+A_{4}(\theta) \xi_{4}(n) e^{-2 \pi i n \theta}\right]+O\left(\frac{1}{n^{2}(\sin \pi \theta)^{5 / 2}}\right),
\end{aligned}
$$

или

$$
\begin{aligned}
\int_{0}^{\theta} & \frac{d \tau}{\sqrt{\cos \pi \tau-\cos \pi \theta}}\left[\sqrt{n} A_{5}(\tau) \xi_{5}(n) e^{2 \pi i n \tau}+\sqrt{n} A_{6}(\tau) \xi_{6}(n) e^{-2 \pi i n \tau}\right. \\
& \left.+\frac{1}{\sqrt{n}} A_{7}(\tau) \xi_{7}(n) e^{2 \pi i n \tau}+\frac{1}{\sqrt{n}} A_{8}(\tau) \xi_{8}(n) e^{-2 \pi i n \tau}\right]+O\left(\frac{1}{|n|^{3 / 2}}\right)
\end{aligned}
$$

uлu

$$
\begin{aligned}
& \frac{1}{\sqrt{\sin \pi \theta}}\left[A_{1}(\theta) \xi_{1}(n) e^{2 \pi i n \theta}+A_{2}(\theta) \xi_{2}(n) e^{-2 \pi i n \theta}+O\left(\frac{1}{n^{2}}\right)\right] \\
& +\frac{1}{\sqrt{\sin \pi \theta}} \int_{\theta}^{1 / 2} \frac{d t}{\sin ^{3 / 2} \pi t} \int_{0}^{t} \frac{d \tau}{\sqrt{\cos \pi \tau-\cos \pi t}}\left[\frac{1}{\sqrt{n}} A_{9}(t) \xi_{9}(n) e^{2 \pi i n(\tau+t-\theta)}\right. \\
& \quad+\frac{1}{\sqrt{n}} A_{10}(t) \xi_{10}(n) e^{-2 \pi i n(\tau+t-\theta)}+n^{-3 / 2} A_{11}(t) \xi_{11}(n) e^{2 \pi i n(\tau+t-\theta)} \\
& \left.+n^{-3 / 2} A_{12}(t) \xi_{12}(n) e^{-2 \pi i n(\tau+t-\theta)}+O\left(\frac{1}{|n|^{5 / 2}}\right)\right]
\end{aligned}
$$

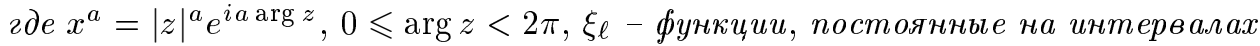
$(0, \infty) u(-\infty, 0),\left|A_{\ell}(\theta)\right| \leqslant C$.

Представления (20)-(22) легко следуют соответственно из (17), (18), (14), если применить формулы Эйлера и произвести элементарные тригонометрические преобразования. Аналогично, (23) следует из сопоставления (15) с (14). 
Лемма 6. Пусть $\gamma \in C^{\infty}(\mathbb{R}), \operatorname{supp} \gamma \subset[-\Delta, \Delta], \theta \in \mathbb{R}, \operatorname{mozдa~}$

$$
\begin{gathered}
\sum_{\ell \in \mathbb{Z}} \gamma\left(2^{-j} \ell\right) e^{2 \pi i \ell \theta}=O\left(\frac{1}{|\sin \pi \theta|}\right) \\
\sum_{\ell \in \mathbb{Z}} \gamma\left(2^{-j} \ell\right) e^{2 \pi i \ell \theta}=O\left(\frac{1}{2^{j}|\sin \pi \theta|^{2}}\right), \\
\int_{0}^{1}\left|\sum_{\ell \in \mathbb{Z}} \gamma\left(2^{-j} \ell\right) e^{2 \pi i \ell \theta}\right| d \theta=O(1) .
\end{gathered}
$$

ДокАЗАТЕльство. Для доказательства (24) применим к левой части преобразование Абеля и воспользуемся явным представлением ядра Дирихле. Для доказательства (25) дважды применим к левой части преобразование Абеля и воспользуемся явным представлением ядра Фейера и свойствами $\gamma$. Левая часть (26) является константой Лебега линейного метода суммирования с множителями суммируемости, определяемыми функцией $\gamma$. Хорошо известно, что в предполагаемых условиях константы Лебега равномерно ограничены.

ЛЕММА 7. В условиях леммы 6

$$
\begin{aligned}
& \int_{0}^{1}\left|\sum_{\ell \in \mathbb{Z}} \gamma\left(2^{-j} \ell\right) e^{2 \pi i \ell \theta}\right| \sqrt{|\sin \pi \theta|} d \theta=O\left(2^{-j / 2}\right), \\
& \int_{0}^{1}\left|\sum_{\ell \in \mathbb{Z}} \gamma\left(2^{-j} \ell\right) e^{2 \pi i \ell \theta}\right| \frac{d \theta}{\sqrt{|\sin \pi \theta|}}=O\left(2^{j / 2}\right) .
\end{aligned}
$$

ДокаЗАТЕльство. Для доказательства (27) разобьем интеграл по схеме:

$$
\int_{0}^{1}=\int_{-1 / 2}^{1 / 2}=\int_{|\theta| \leqslant 2^{-j}}+\sum_{s=1}^{j-1} \int_{2^{-j+s-1} \leqslant|\theta| \leqslant 2^{-j+s}}=\sum_{s=0}^{j-1} I_{s} .
$$

Из (26) следует, что $I_{0}=O\left(2^{-j / 2}\right)$. При $s>0$, применяя (25) к подынтегральному выражению, имеем

$$
\sum_{s=1}^{j-1} I_{s} \leqslant C 2^{-j} \sum_{s=1}^{j-1} 2^{\frac{j-s}{2}}=O\left(2^{-j / 2}\right) .
$$

Аналогично, используя (24) вместо (25) и очевидное соотношение

$$
\sum_{\ell \in \mathbb{Z}} \gamma\left(2^{-j} \ell\right) e^{2 \pi i \ell \theta}=O\left(2^{j}\right)
$$

вместо (26), получаем (28).

Введем обозначение $e_{\tau}=\{t \in \mathbb{R}:|\sin \pi t| \leqslant|\sin \pi \tau|\}$. Для фиксированного $\phi \in[0,1 / 2]$ положим $g_{1}(t)=\chi_{e_{2 \phi}}(t) \cos \pi t$, где $\chi_{e}-$ характеристическая функция множества $e, g_{2}(t)=\cos \pi t-g_{1}(t)$. 
Лемма 8. Пусть $\gamma \in C^{\infty}(\mathbb{R}), \operatorname{supp} \gamma \subset[-\Delta, \Delta] \backslash[-\delta, \delta], \tau \in \mathbb{R}$,

$$
\sigma_{\alpha, \nu}^{ \pm}(\theta)=\sum_{\ell \in \mathbb{Z}} \gamma\left(2^{-j} \ell\right)\left(2^{-j} \ell\right)^{\alpha} \xi_{\nu}\left(2^{-j} \ell\right) e^{2 \pi i \ell(\tau \pm \theta)}
$$

где $\alpha \in \mathbb{R}, \xi_{\nu}-$ функции из леммы 5. Тогда

$$
\begin{aligned}
& \int_{0}^{1}\left|\sum_{\ell \in \mathbb{Z}} \gamma\left(2^{-j} \ell\right) e^{2 \pi i \ell \tau} X_{\ell}(\cos \pi \theta)\right| d g_{1}(\theta) \\
& \leqslant C \sqrt{\phi}\left\{\int_{-1 / 2}^{1 / 2}\left(\left|\sigma_{0,1}^{+}(\theta)\right|+\left|\sigma_{0,2}^{-}(\theta)\right|\right) d \theta+O(1)\right\} \\
& \int_{0}^{1}\left|\sum_{\ell \in \mathbb{Z}} \gamma\left(2^{-j} \ell\right) e^{2 \pi i \ell \tau} X_{\ell}(\cos \pi \theta)\right| d g_{2}(\theta) \\
& \leqslant C \int_{-1 / 2}^{1 / 2}\left(\left|\sigma_{0,1}^{+}(\theta)\right|+\left|\sigma_{0,2}^{-}(\theta)\right|\right) \frac{\left|g_{2}^{\prime}(\theta)\right| d \theta}{\sqrt{|\sin \pi \theta|}} \\
& \quad+C 2^{-j} \int_{-1 / 2}^{1 / 2}\left(\left|\sigma_{-1,3}^{-}(\theta)\right|+\left|\sigma_{-1,4}^{-}(\theta)\right|\right) \frac{\left|g_{2}^{\prime}(\theta)\right| d \theta}{\sqrt{|\sin \pi \theta|^{3}}}+O\left(2^{-j / 2}\right) .
\end{aligned}
$$

ДоКАЗАТЕЛЬСТво. Соотношение (29) несложно вьвести из (20), если при оценке остаточного члена принять во внимание структуру носителя функции $\gamma$ и учесть периодичность функций $\sigma_{\alpha, \nu}^{ \pm}$. Для доказательства (30) разобьем интеграл в левой части по схеме:

$$
\int_{[0,1] \backslash e_{2 \phi}}=\int_{\left(e_{2}-j \backslash e_{2 \phi}\right) \cap[0,1]}+\int_{[0,1] \backslash e_{2}-j}=I_{1}+I_{2} .
$$

Если $\phi \geqslant 2^{-j-1}$, то $I_{1}=0$. Если $\phi \leqslant 2^{-j-1}$, то те же рассуждения, которые применялись для доказательства (29), дают

$$
I_{1} \leqslant C\left\{\int_{-1 / 2}^{1 / 2}\left(\left|\sigma_{0,1}^{+}(\theta)\right|+\left|\sigma_{0,2}^{-}(\theta)\right|\right) \frac{\left|g_{2}^{\prime}(\theta)\right| d \theta}{\sqrt{|\sin \pi \theta|}}+O\left(2^{-j / 2}\right)\right\} .
$$

Аналогично, чтобы оценить $I_{2}$, применим (21). Снова учитывая структуру носителя $\gamma$ при оценке остатка, получаем (30).

ЛЕмма 9. Пусть $\mu$ - ограниченная монотонно убъвающая суммируемая на $[0, \infty)$ функиия. Тогда для всех $x, y \in \mathbb{R}$

$$
\sum_{k \in \mathbb{Z}} \mu(|x+k|) \mu(|y+k|) \leqslant A \mu\left(\frac{|x-y|}{4}\right),
$$

әде $A$ - постоянная, зависящая только от $\mu$.

Эта несложная лемма известна, доказательство несколько более общего утверждения приводится, например, в [12].

Пусть $\psi \in L(\mathbb{R}), \alpha \in \mathbb{R}, \nu=1, \ldots, 8$. Положим

$$
s_{n, \alpha, \nu}^{ \pm}(\tau)=s_{n, \alpha, \nu}^{ \pm}(\tau, \psi)=\sum_{\ell \in \mathbb{Z}} e^{2 \pi i\left(n 2^{-j} \pm \tau\right) \ell}\left(2^{-j} \ell\right)^{\alpha} \xi_{\nu}\left(2^{-j} \ell\right) \widetilde{\psi}\left(2^{-j} \ell\right),
$$

где $\widetilde{\psi}$ - преобразование Фурье функции $\psi, \xi_{\nu}$ - функции из леммы 5. 
Лемма 10. Пусть $\widetilde{\psi} \in C^{\infty}, \operatorname{supp} \widetilde{\psi} \subset[-\Delta, \Delta] \backslash[-\delta, \delta], \alpha, \beta \in \mathbb{R},|\tau| \leqslant 1 / 4$, $|\gamma| \leqslant 1 / 2, \operatorname{mогда~}$

$$
I=\int_{-1 / 2}^{1 / 2} \sum_{n=0}^{2^{j}-1}\left|s_{n, \alpha, \nu}^{ \pm}(\tau)\right|\left|s_{n, \beta, \mu}^{ \pm}(\theta \pm \tau)\right||\sin \pi \theta|^{\gamma} d \theta=O\left(2^{(1-\gamma) j}\right)
$$

где знаки “+” или “-” в выражениях $s_{n, \beta, \mu}^{ \pm} u \theta \pm \tau$ согласованы таким образом, что $\tau$ входит во все әкспоненты подынтегрального выражения $c$ одним и тем же знаком.

ДокАЗАТЕЛЬСтво. Не уменьшая общности, можно ограничиться рассмотрением знаков “+” в качестве каждого из трех “土”. Кроме того, оставим только первую половину слагаемых в подынтегральной сумме. Для второй половины оценка проводится с помощью аналогичных рассуждений. Функции $\psi_{\alpha, \nu}, \psi_{\beta, \mu}$, определяемые равенствами $\widetilde{\psi}_{\alpha, \nu}(u)=\widetilde{\psi}(u) \xi_{\nu}(u)(u)^{\alpha}, \widetilde{\psi}_{\beta, \mu}(u)=\widetilde{\psi}(u) \xi_{\mu}(u)(u)^{\beta}$, очевидно, обладают теми же свойствами, что и $\psi$, поэтому

$$
\left|\psi_{\alpha, \nu}(u)\right|,\left|\psi_{\beta, \mu}(u)\right|=O\left(\frac{1}{1+u^{2}}\right)
$$

и по формуле суммирования Пуассона

$$
\begin{aligned}
& \sum_{n=0}^{2^{j-1}-1}\left|s_{n, \alpha, \nu}^{+}(\tau)\right|\left|s_{n, \beta, \mu}^{+}(\theta+\tau)\right| \\
& \quad=2^{2 j} \sum_{n=0}^{2^{j-1}-1}\left|\sum_{\ell \in \mathbb{Z}} \psi_{\alpha, \nu}\left(2^{j} \tau+2^{j} \ell+n\right)\right|\left|\sum_{k \in \mathbb{Z}} \psi_{\beta, \mu}\left(2^{j}(\theta+\tau)+2^{j} k+n\right)\right| .
\end{aligned}
$$

Поскольку в силу (31)

$$
\sum_{\ell \neq 0}\left|\psi_{\alpha, \nu}\left(2^{j} \tau+2^{j} \ell+n\right)\right|=O\left(2^{-2 j}\right), \quad \sum_{n=0}^{2^{j-1}-1} \sum_{k \in \mathbb{Z}}\left|\psi_{\beta, \mu}\left(2^{j}(\theta+\tau)+2^{j} k+n\right)\right|=O(1),
$$

правая часть (32) не превосходит

$$
2^{2 j} \sum_{n=0}^{2^{j-1}-1} \sum_{k \in \mathbb{Z}}\left|\psi_{\alpha, \nu}\left(2^{j} \tau+n\right)\right|\left|\psi_{\beta, \mu}\left(2^{j}(\theta+\tau)+2^{j} k+n\right)\right|+O(1) .
$$

Отсюда, из леммы 9 и (32) следует

$$
\begin{aligned}
& \sum_{n=0}^{2^{j-1}-1}\left|s_{n, \alpha, \nu}^{+}(\tau)\right|\left|s_{n, \beta, \mu}^{+}(\theta+\tau)\right||\sin \pi \theta|^{\gamma} \\
& \quad=O\left(1+\sum_{k \in \mathbb{Z}} \frac{2^{2 j}|\theta|^{\gamma}}{1+\left(2^{j}(\theta+k)\right)^{2}}\right)=O\left(1+\frac{2^{2 j}|\theta|^{\gamma}}{1+\left(2^{j}(\theta)\right)^{2}}\right)
\end{aligned}
$$


Разбивая интеграл $I$ по схеме

$$
\int_{-1 / 2}^{1 / 2}=\int_{|\theta| \leqslant 2^{-j}}+\sum_{s=0}^{j-1} \int_{2^{-j+s-1} \leqslant|\theta| \leqslant 2^{-j+s}}
$$

и применяя (33) к подынтегральному выражению в каждом слагаемом, получаем

$$
I=O\left(2^{j(1-\gamma)}+2^{j(1-\gamma)} \sum_{s=0}^{j-1} 2^{-s(1-\gamma)}\right)=O\left(2^{j(1-\gamma)}\right) .
$$

\section{СлЕДСТВИЕ 11. В условиях леммы 10 выполняется соотношение}

$$
\int_{-1 / 2}^{1 / 2} \sum_{n=0}^{2^{j}-1}\left|s_{n, \alpha, \nu}^{ \pm}(\tau)\right|\left|s_{n, \beta, \mu}^{ \pm}(\theta)\right| d \theta=O\left(2^{j}\right) .
$$

Утверждение следствия является частньм случаем леммы 10 , если произвести замену переменной $\theta=\theta^{\prime} \pm \tau$ и принять во внимание периодичность подынтегральной функции.

Лемма 12. Пусть $\psi, H_{j}$ удовлетворяют условиям леммы 10, $\alpha \in \mathbb{R}$. Тогда

$$
\begin{aligned}
& \int_{0}^{1} \sum_{n=0}^{2^{j}-1}\left|s_{n, \alpha, \nu}^{ \pm}(\tau)\right|\left|S_{j}^{n} H_{j}(\cos \pi \theta)\right| d g_{1}(\theta) \\
& \leqslant C \sqrt{\phi}\left\{2^{-j / 2} \int_{-1 / 2}^{1 / 2} \sum_{n=0}^{2^{j}-1}\left|s_{n, \alpha, \nu}^{ \pm}(\tau)\right|\left(\left|s_{n, 0,1}^{+}(\theta)\right|+\left|s_{n, 0,2}^{-}(\theta)\right|\right) d \theta+O\left(2^{j / 2}\right)\right\} \\
& \int_{0}^{1} \sum_{n=0}^{2^{j}-1}\left|s_{n, \alpha, \nu}^{ \pm}(\tau)\right|\left|S_{j}^{n} H_{j}(\cos \pi \theta)\right| d g_{2}(\theta) \\
& \leqslant C 2^{-j / 2} \int_{-1 / 2}^{1 / 2} \sum_{n=0}^{2^{j}-1}\left|s_{n, \alpha, \nu}^{ \pm}(\tau)\right|\left(\left|s_{n, 0,1}^{+}(\theta)\right|+\left|s_{n, 0,2}^{-}(\theta)\right|\right) \frac{\left|g_{2}^{\prime}(\theta)\right| d \theta}{\sqrt{|\sin \pi \theta|}} \\
& +C 2^{-3 j / 2} \int_{-1 / 2}^{1 / 2} \sum_{n=0}^{2^{j}-1}\left|s_{n, \alpha, \nu}^{ \pm}(\tau)\right|\left(\left|s_{n,-1,3}^{+}(\theta)\right|+\left|s_{n,-1,4}^{-}(\theta)\right|\right) \frac{\left|g_{2}^{\prime}(\theta)\right| d \theta}{\sqrt{|\sin \pi \theta|^{3}}}+O(1)
\end{aligned}
$$

ДоКАЗАТЕЛЬСТво. На основании (13) и (20), (21), принимая во внимание, что $\widetilde{\psi}\left(2^{-j} \ell\right)$ отлично от нуля лишш при $2^{j} \delta \leqslant|\ell| \leqslant 2^{j} \Delta$, имеем

$$
\begin{aligned}
\left|S_{j}^{n} H_{j}(\cos \pi \theta)\right| \leqslant & C\left(\frac{2^{-j}}{\sin \pi \theta}\right)^{1 / 2}\left(\left|s_{n, 0,1}^{+}(\theta)\right|+\left|s_{n, 0,2}^{-}(\theta)\right|\right)+O\left(\frac{2^{-j / 2}}{\sin ^{3 / 2} \pi \theta}\right), \\
\left|S_{j}^{n} H_{j}(\cos \pi \theta)\right| \leqslant & C\left(\frac{2^{-j}}{\sin \pi \theta}\right)^{1 / 2}\left(\left|s_{n, 0,1}^{+}(\theta)\right|+\left|s_{n, 0,2}^{-}(\theta)\right|\right) \\
& +C\left(\frac{2^{-j}}{\sin \pi \theta}\right)^{3 / 2}\left(\left|s_{n,-1,3}^{+}(\theta)\right|+\left|s_{n,-1,4}^{-}(\theta)\right|\right)+O\left(\frac{2^{-3 j / 2}}{\sin ^{5 / 2} \pi \theta}\right) .
\end{aligned}
$$


Пусть $\psi_{\alpha, \nu}$ - функция, определенная в лемме 10 . Из формулы суммирования Пуассона и (31) следует

$$
\sum_{n=0}^{2^{j}-1}\left|s_{n, \alpha, \nu}^{ \pm}(\tau)\right|=2^{j} \sum_{n=0}^{2^{j}-1}\left|\sum_{\ell \in \mathbb{Z}} \psi_{\alpha, \nu}\left( \pm 2^{j} \tau+2^{j} \ell+n\right)\right|=O\left(2^{j}\right)
$$

Соотношения (36) и (38) влекут (34), если принять во внимание периодичность функций $s_{n, \eta, \varkappa}^{ \pm}(\theta)$. Для доказательства (35) разобьем интеграл в левой части по схеме

$$
\int_{[0,1] \backslash e_{2 \phi}}=\int_{\left(e_{2}-j \backslash e_{2 \phi}\right) \cap[0,1]}+\int_{[0,1] \backslash e_{2}-j}=I_{1}+I_{2} .
$$

Если $|\phi| \geqslant 2^{-j-1}$, то $I_{1}=0$. Если $|\phi| \leqslant 2^{-j-1}$, то те же рассуждения, которые применялись для доказательства $(34)$, дают

$$
I_{1} \leqslant C 2^{-j / 2} \int_{-1 / 2}^{1 / 2} \sum_{n=0}^{2^{j}-1}\left|s_{n, \alpha, \nu}^{ \pm}(\tau)\right|\left(\left|s_{n, 0,1}^{+}(\theta)\right|+\left|s_{n, 0,2}^{-}(\theta)\right|\right) \frac{\left|g_{2}^{\prime}(\theta)\right| d \theta}{\sqrt{|\sin \pi \theta|}}+O(1) .
$$

Аналогично, чтобы оценить $I_{2}$, применим (37) и (38). Осталось заметить, что

$$
2^{-j / 2} \int_{[0,1] \backslash e_{2}-j} \frac{d g_{2}(\theta)}{\sin ^{5 / 2} \pi \theta}=O(1)
$$

Лемма 13. Пусть функиии $\psi, H_{j}$ удовлетворяют условиям леммъ 10, $n=0, \ldots, 2^{j}-1, \operatorname{moгдa~}$

$$
\int_{0}^{1}\left|S_{j}^{n} H_{j}(\cos \pi \theta)\right| d \cos \pi \theta=O\left(2^{-j / 2}\right)
$$

ДокАЗАТЕльство. На основании (36) имеем

$$
\int_{0}^{1}\left|S_{j}^{n} H_{j}(\cos \pi \theta)\right| d \cos \pi \theta \leqslant C 2^{-j / 2} \int_{-1 / 2}^{1 / 2}\left(\left|s_{n, 0,1}^{+}(\theta)\right|+\left|s_{n, 0,2}^{-}(\theta)\right|\right) d \theta+O\left(2^{-j / 2}\right) .
$$

Пусть $\psi_{0, \nu}, \nu=1,2,-$ функции, определенные в лемме 10 . По формуле суммирования Пуассона

$$
\int_{-1 / 2}^{1 / 2}\left|s_{n, 0, \nu}^{ \pm}(\theta)\right| d \theta \leqslant 2^{j} \sum_{\ell \in \mathbb{Z}} \int_{-1 / 2}^{1 / 2}\left|\psi_{0, \nu}\left( \pm 2^{j} \theta+2^{j} \ell+n\right)\right| d \theta=\int_{-\infty}^{\infty}\left|\psi_{0, \nu}( \pm \theta)\right| d \theta
$$

Осталось заметить, что интеграл в правой части этого равенства конечен в силу (31). 
Лемма 14.

$$
\int_{-1}^{1}\left|\frac{1}{N} \sum_{n=0}^{N} \sum_{k=0}^{2 n} P_{k}(x) P_{k}(t)\right| d t=O(1)
$$

ДокаЗАТЕЛьство. Поскольку функции Лебега сумм Фейера-Лежандра равномерно ограничены (см., например, [13]), в силу равенства

$$
\begin{aligned}
& \sum_{n=0}^{2 N} \sum_{k=0}^{n} P_{k}(x) P_{k}(t) \\
& \quad=\sum_{n=0}^{N} \sum_{k=0}^{2 n} P_{k}(x) P_{k}(t)+\sum_{n=0}^{N-1} \sum_{k=0}^{2 n+1} P_{k}(x) P_{k}(t) \\
& \quad=2 \sum_{n=0}^{N-1} \sum_{k=0}^{2 n} P_{k}(x) P_{k}(t)+\sum_{k=0}^{2 N} P_{k}(x) P_{k}(t)+\sum_{n=0}^{N-1} P_{2 n+1}(x) P_{2 n+1}(t) \\
& \quad=2 \sum_{n=0}^{N} \sum_{k=0}^{2 n} P_{k}(x) P_{k}(t)-\sum_{n=0}^{N} P_{2 n}(x) P_{2 n}(t)
\end{aligned}
$$

достаточно доказать соотношение

$$
I=\frac{1}{N} \int_{-1}^{1}\left|\sum_{n=0}^{N} P_{2 n}(x) P_{2 n}(t)\right| d t=O(1)
$$

Положим $t=\cos \pi \theta, x=\cos \pi \phi, \phi, \theta \in[0,1]$. Поскольку $P_{2 n}$ - четная функция, достаточно ограничиться рассмотрением случая $\phi \in[0,1 / 2]$. Применяя (17) к $P_{2 n}(\cos \pi \theta)$ и используя (19) при оценке остаточного члена, имеем

$$
I=\frac{\sqrt{2}}{N \sqrt{\pi}} \int_{0}^{1}\left|\sum_{n=0}^{N} P_{2 n}(\cos \pi \phi) \cos \left[\left(2 n+\frac{1}{2}\right) \pi \theta-\frac{\pi}{4}\right]\right| \frac{d \cos \pi \theta}{\sqrt{\sin \pi \theta}}+O(1) .
$$

Интеграл в правой части разобьем по схеме

$$
\int_{0}^{1} d \cos \pi \theta=\int_{0}^{1} d g_{1}(\theta)+\int_{0}^{1} d g_{2}(\theta)=I_{1}+I_{2}
$$

Для оценки $I_{2}$ при $\phi \neq 0$ применим к $P_{2 n}(\cos \pi \phi)$ формулу Дирихле-Мелера (14). После перестановки интегралов получим

$$
\begin{aligned}
I_{2} \leqslant & \frac{1}{2} \int_{0}^{\phi} \frac{d \tau}{\sqrt{\cos \pi \tau-\cos \pi \phi}} \int_{0}^{1}\left\{\left|\sum_{n=0}^{N} e^{2 \pi i n(\theta+\tau)} \sqrt{n+\frac{1}{4}}\right|\right. \\
& +\left|\sum_{n=0}^{N} e^{2 \pi i n(\theta-\tau)} \sqrt{n+\frac{1}{4}}\right|+\left|\sum_{n=0}^{N} e^{-2 \pi i n(\theta+\tau)} \sqrt{n+\frac{1}{4}}\right| \\
& \left.+\left|\sum_{n=0}^{N} e^{-2 \pi i n(\theta-\tau)} \sqrt{n+\frac{1}{4}}\right|\right\} \frac{d g_{2}(\theta)}{\sqrt{\sin \pi \theta}}
\end{aligned}
$$


Производя преобразование Абеля в каждой из сумм и используя хорошо известные свойства ядер Дирихле, устанавливаем, что эти суммы имеют порядок $O(\sqrt{N} /|\sin \pi(\theta \pm \tau)|)$. Таким образом, внутренний интеграл в (40) не превосходит суммы четырех интегралов вида

$$
C \sqrt{N} \int_{0}^{1} \frac{d g_{2}(\theta)}{|\sin \pi(\theta \pm \tau)| \sqrt{\sin \pi \theta}}
$$

Поскольку $\tau \leqslant \phi$, то $1 / \sin |\pi(\theta \pm \tau)| \leqslant C / \sin \pi \theta$ при $\theta \geqslant 2 \phi$. Следовательно,

$$
\int_{0}^{1} \frac{d g_{2}(\theta)}{|\sin \pi(\theta \pm \tau)| \sqrt{\sin \pi \theta}} \leqslant C \int_{0}^{1} \frac{d \theta}{\sqrt{\sin \pi \theta}}=O(1)
$$

Сопоставляя это соотношение с (41) и (40), получаем

$$
I_{2}=O(\sqrt{N})
$$

Для $\phi=0$ интеграл $I_{2}$ можно оценить аналогично, используя вместо формулы Дирихле-Мелера равенство (19). Теперь оценим $I_{1}$ в случае, когда $\phi \leqslant 1 / N$. Для $\phi \neq 0$ снова воспользуемся формулой Дирихле-Мелера:

$$
I_{1} \leqslant C \int_{0}^{\phi} \frac{d \tau}{\sqrt{\cos \pi \tau-\cos \pi \phi}} \int_{0}^{1} \sum_{n=0}^{N} \sqrt{n+\frac{1}{4}} \frac{g_{1}^{\prime}(\theta)}{\sqrt{\sin \pi \theta}} d \theta .
$$

Учитывая, что $g_{1}^{\prime}(\theta) / \sqrt{\sin \pi \theta} \leqslant C \sqrt{\phi} \leqslant C / \sqrt{N}$, получаем

$$
I_{1}=O(N)
$$

Точно так же (43) следует из (19) при $\phi=0$. Наконецрассмотрим случай $\phi \geqslant 1 / N$. Сопоставляя соотношения (14) и (15), имеем

$$
\begin{aligned}
& (\sin \pi \phi)^{1 / 2} P_{2 n}(\cos \pi \phi)=\sqrt{\frac{2}{\pi}} \cos \left[\left(2 n+\frac{1}{2}\right) \pi \phi-\frac{\pi}{4}\right] \\
& -\frac{\sqrt{2 \pi}}{\sqrt{2 n+\frac{1}{2}}} \int_{\phi}^{1 / 2} \frac{\sin \left[\pi\left(2 n+\frac{1}{2}\right)(t-\phi)\right]}{4 \sin ^{3 / 2} \pi t} d t \int_{0}^{t} \frac{\cos \left[\pi\left(2 n+\frac{1}{2}\right) \tau\right]}{\sqrt{\cos \pi \tau-\cos \pi t}} d \tau+O\left(\frac{1}{n^{2}}\right) .
\end{aligned}
$$

Отсюда, принимая во внимание, что $g_{1}^{\prime}(\theta) / \sqrt{\sin \pi \theta} \leqslant C \sqrt{\phi}$ и $\phi^{-1} \leqslant N$, получаем

$$
I_{1} \leqslant C \int_{\phi}^{1 / 2} \frac{d t}{t^{3 / 2}} \sum_{n=0}^{N} \frac{1}{\sqrt{n}}+O(1)=O\left(\sqrt{\frac{N}{\phi}}+1\right)=O(N) .
$$

Эта оценка вместе с (42) и (43) дает (39). 


\section{§5. Доказательство теоремы 1}

Докажем, что система ортогональных многочленов $T_{n}$, построенная в $\S 3$, является базисом Шаудера в пространстве $C[-1,1]$. Каждой функции $f \in C[-1,1]$ сопоставим ее ряд Фурье по системе $T_{n}$, через $\sigma_{N}(f)$ обозначим частичную сумму этого ряда порядка $N$. Если $N=2^{j}+m 2^{j-s}+r, m=0, \ldots, 2^{s}-1$, $r=0, \ldots, 2^{j-s}-1$, то

$$
\begin{aligned}
\sigma_{N}(f)=\sum_{n=1}^{N}\left\langle f, T_{n}\right\rangle T_{n}= & \sum_{n=1}^{2^{j}}\left\langle f, T_{n}\right\rangle T_{n}+\sum_{k=0}^{m-1} \sum_{n=0}^{2^{j-s}-1}\left\langle f, S_{j-s}^{n} H_{j}^{s, k+1}\right\rangle S_{j-s}^{n} H_{j}^{s, k+1} \\
& +\sum_{n=0}^{r}\left\langle f, S_{j-s}^{n} H_{j}^{s, m+1}\right\rangle S_{j-s}^{n} H_{j}^{s, m+1} .
\end{aligned}
$$

Первая сумма в правой части является ортогональной проекцией функции $f$ на пространство $V_{j}$. Переразложенная по ортонормированному базису $S_{j}^{n} G_{j}$, $n=0, \ldots, 2^{j}-1$, она примет вид

$$
\sum_{n=0}^{2^{j}-1}\left\langle f, S_{j}^{n} G_{j}\right\rangle S_{j}^{n} G_{j} .
$$

Пусть $|\nu| \leqslant 2^{j-2}$, из построения функций $G_{j}, H_{j}^{s, k}$ следует, что $\widehat{H}_{j}^{s, k}(\nu)=0$, $k=1, \ldots, 2^{s}, \widehat{G}_{j}(\nu)=2^{-j / 2}, \widehat{G}_{j}\left(\nu+2^{j} n\right)=0, n \in \mathbb{Z}_{+}$, поэтому

$$
\begin{aligned}
\sigma_{N}\left(X_{\nu}\right) & =\sum_{n=0}^{2^{j}-1}\left\langle X_{\nu}, S_{j}^{n} G_{j}\right\rangle S_{j}^{n} G_{j} \\
& =\sum_{\ell \in \mathbb{Z}} \widehat{G}_{j}(\ell)\left\langle X_{\nu}, X_{\ell}\right\rangle \sum_{k \in \mathbb{Z}} \widehat{G}_{j}(k) X_{k} \sum_{n=0}^{2^{j}-1} e^{2 \pi i 2^{-j} n(k-\ell)}
\end{aligned}
$$

Из этого равенства, ортонормированности системы $\left\{X_{k}\right\}_{k \in \mathbb{Z}}$ и (4) следует $\sigma_{N}\left(X_{\nu}\right)=X_{\nu}$. Таким образом, оператор $\sigma_{N}$ не изменяет алгебраические многочлены степени не выше $2^{j-1}$, что влечет

$$
\left\|f-\sigma_{N}(f)\right\| \leqslant\left\|\sigma_{N}\right\|_{C \rightarrow C} E_{2^{j-1}}(f)
$$

где $E_{m}(f)$ - величина наилучшего приближения функции $f$ алгебраическими многочленами порядка $m$. Для доказательства теоремы осталось проверить ограниченность последовательности $\left\|\sigma_{N}\right\|_{C \rightarrow C}$. Для этого достаточно убедиться в равномерной (по $x \in[-1,1])$ ограниченности интегралов

$$
\begin{aligned}
J(x) & =\int_{-1}^{1}\left|\sum_{n=0}^{2^{j}-1} S_{j}^{n} G_{j}(x) \overline{S_{j}^{n} G_{j}(t)}\right| d t \\
J^{m}(x) & =\int_{-1}^{1} \sum_{n=0}^{2^{j-s}-1}\left|S_{j-s}^{n} H_{j}^{s, m}(x) S_{j-s}^{n} H_{j}^{s, m}(t)\right| d t, \quad m=1, \ldots, 2^{s} .
\end{aligned}
$$


Зафиксируем $m$ и займемся оценкой $J^{m}$. Переобозначим функции $H_{j}^{s, m}, \psi^{s, m}$ через $H_{j}, \psi$ (выше эти обозначения имели другой смысл, но они уже больше не будут использоваться). Отметим, что функция $\widetilde{\psi}$ удовлетворяет условиям леммы 10. Ясно, что

$$
J^{m}(x) \leqslant \int_{-1}^{1} \sum_{n=0}^{2^{j}-1}\left|S_{j}^{n} H_{j}(x) S_{j}^{n} H_{j}(t)\right| d t=I .
$$

Ограничимся рассмотрением случая $x \in[0,1]$, для $x \in[-1,0]$ доказательство аналогично. Положим $x=\cos \pi \phi, \phi \in[0,1 / 2]$. Пусть сначала $\phi \in[1 / 4,1 / 2]$. Применяя к $S_{j}^{n} H_{j}(\cos \pi \phi)$ неравенства (36) и $(38)$, имеем

$$
I \leqslant C 2^{j / 2} \max _{n} \int_{-1}^{1}\left|S_{j}^{n} H_{j}(t)\right| d t
$$

Отсюда и из леммы 13 следует $I=O(1)$. Теперь рассмотрим случай $\phi \in(0,1 / 4]$. Произведем в интеграле $I$ замену переменной $t=\cos \pi \theta, \theta \in[0,1]$, и разобьем его по схеме

$$
I=\int_{0}^{1} d g_{1}(\theta)+\int_{0}^{1} d g_{2}(\theta)=I_{1}+I_{2}
$$

При $\phi \geqslant 2^{-j}$ для доказательства ограниченности $I_{1}$ оценим $S_{j}^{n} H_{j}(\cos \phi)$, используя (23) и учитывая структуру носителя функции $\widetilde{\psi}$ :

$$
\begin{aligned}
& \left|S_{j}^{n} H_{j}(\cos \phi)\right| \leqslant C \frac{2^{-j / 2}}{\sqrt{\phi}}\left[\left|s_{n, 0,1}^{+}(\phi)\right|+\left|s_{n, 0,2}^{-}(\phi)\right|\right. \\
& \quad+2^{-j / 2} \int_{\phi}^{1 / 2} \frac{d t}{t^{3 / 2}} \int_{0}^{t} \frac{d \tau}{\sqrt{\cos \pi \tau-\cos \pi t}} \\
& \left.\quad \times\left(\left|s_{n,-1 / 2,9}^{+}(\tau+t-\phi)\right|+\left|s_{n,-1 / 2,10}^{-}(\tau+t-\phi)\right|\right)+O\left(2^{-j / 2}\right)\right] .
\end{aligned}
$$

В каждом слагаемом внеинтегральной и подынтегральной сумм присутствует выражение вида $s_{n, \alpha, \nu}^{ \pm}(u)$. Соответствуюшее слагаемоев $I_{1}$ оценится через интеграл

$$
\int_{0}^{1} \sum_{n=0}^{2^{j}-1}\left|s_{n, \alpha, \nu}^{ \pm}(u)\right|\left|S_{j}^{n} H_{j}(\cos \pi \theta)\right| d g_{1}(\theta)=I_{1}^{\alpha, \nu}
$$

Применяя к этому интегралу неравенство (34), а затем к каждому слагаемому правой части (34) следствие 11 , устанавливаем, что $I_{1}^{\alpha, \nu} \leqslant C 2^{j / 2} \sqrt{\phi}$. Отсюда, учитывая при оценке остаточного члена лемму 13, получаем

$$
I_{1}=O\left(1+\left(2^{j} \phi\right)^{-1 / 2}\right)=O(1) .
$$

Для доказательства ограниченности $I_{2}$ при $\phi \neq 0$ оценим $S_{j}^{n} H_{j}(\cos \phi)$, используя (22):

$$
\begin{aligned}
\left|S_{j}^{n} H_{j}(\cos \phi)\right| \leqslant & C \int_{0}^{\phi} \frac{d t}{\sqrt{\cos \pi t-\cos \pi \phi}}\left(\left|s_{n, 1 / 2,5}^{+}(t)\right|+\left|s_{n, 1 / 2,6}^{-}(t)\right|\right. \\
& \left.+\left|s_{n,-1 / 2,7}^{+}(t)\right|+\left|s_{n,-1 / 2,8}^{-}(t)\right|+O\left(2^{-j / 2}\right)\right) .
\end{aligned}
$$


В каждом слагаемом подынтегральной суммы присутствует выражение вида $s_{n, \alpha, \nu}^{ \pm}(t)$. Соответствующее слагаемое в $I_{2}$ оценивается через интеграл

$$
\int_{0}^{1} \sum_{n=0}^{2^{j}-1}\left|s_{n, \alpha, \nu}^{ \pm}(t)\right|\left|S_{j}^{n} H_{j}(\cos \pi \theta)\right| d g_{2}(\theta)=I_{2}^{\alpha, \nu} .
$$

Применим к этому интегралу неравенство (35) и произведем в каждом слагаемом правой части (35) замену переменной $\theta^{\prime}=\theta \pm t$, выбирая знак “+” или “-" так, чтобы $t$ входило во все экспоненты подынтегрального выражения с одним и тем же знаком. Учитывая, что при $t \leqslant \phi$

$$
\left|g_{2}^{\prime}(\theta)\right||\sin \pi \theta|^{-1 \pm 1 / 2} \leqslant C\left|\sin \pi \theta^{\prime}\right|^{ \pm 1 / 2},
$$

на основании леммы 10 при $\gamma= \pm 1 / 2$ получаем $I_{2}^{\alpha, \nu}=O(1)$. Отсюда с учетом леммы 13 при оценке остаточного члена устанавливаем ограниченность $I_{2}$. Теперь оценим $I_{1}$ при $\phi \in\left(0,2^{-j}\right)$. Те же рассуждения, что и для случая $\phi \geqslant 2^{-j}$, дают

$$
\int_{0}^{1} \sum_{n=0}^{2^{j}-1}\left|s_{n, \alpha, \nu}^{ \pm}(u)\right|\left|S_{j}^{n} H_{j}(\cos \pi \theta)\right| d g_{1}(\theta)=O\left(2^{j / 2} \sqrt{\phi}\right) .
$$

Сопоставляя это с (45), учитывая, что $\sqrt{\phi}=O\left(2^{-j / 2}\right)$, и принимая во внимание лемму 13 , опять получаем $I_{1}=O(1)$. При $\phi=0$ интеграл $I_{1}$ отсутствует, а $I_{2}$ можно оценить по той же схеме, используя вместо (22) следующее равенство

$$
X_{\ell}(1)=\sqrt{2 i \ell}+\frac{1}{4 \sqrt{2 i \ell}}+O\left(\frac{1}{|\ell|^{3 / 2}}\right),
$$

где $\sqrt{z}=\sqrt{|z|} e^{i \frac{\arg z}{2}},-\pi \leqslant \arg z \leqslant \pi$. Таким образом, доказана ограниченность $J^{m}$.

Для оценки $J$ преобразуем подынтегральное выражение:

$$
\begin{aligned}
& \sum_{n=0}^{2^{j}-1} \overline{S_{j}^{n} G_{j}(t)} S_{j}^{n} G_{j}(x) \\
& \quad=2^{-j} \sum_{\ell \in \mathbb{Z}} \widetilde{\varphi}\left(2^{-j} \ell\right) \overline{X_{\ell}(t)} \sum_{k \in \mathbb{Z}} \widetilde{\varphi}\left(2^{-j} k\right) X_{k}(x) \sum_{n=0}^{2^{j}-1} e^{2 \pi i 2^{-j} n(k-\ell)} \\
& \quad=\sum_{m \in \mathbb{Z}} \sum_{\ell \in \mathbb{Z}} \widetilde{\varphi}\left(2^{-j} \ell\right) \overline{X_{\ell}(t)} \widetilde{\varphi}\left(2^{-j} \ell+m\right) X_{\ell+2^{j} m}(x)=\sum_{m \in \mathbb{Z}} \omega_{m}(t, x) .
\end{aligned}
$$

Поскольку $\operatorname{supp} \widetilde{\varphi} \subset\left[-\frac{1}{2}(1+\varepsilon), \frac{1}{2}(1+\varepsilon)\right]$, то $\omega_{m}(t, x)=0$ для всех $m \neq 0,1,-1$. Покажем, что

$$
\int_{-1}^{1}\left|\omega_{m}(t, x)\right| d t=O(1)
$$

для $m=0,1,-1$. Перепишем $\omega_{0}(t, x)$ в вешественном виде:

$$
\omega_{0}(t, x)=1+\sum_{n=1}^{\infty} \widetilde{\varphi}^{2}\left(2^{-j} n\right)\left(P_{2 n-1}(x) P_{2 n-1}(t)+P_{2 n}(x) P_{2 n}(t)\right) .
$$

После двукратного применения преобразования Абеля к этой сумме на основании леммы 14 получаем (48) для $m=0$. 
Пусть $\theta, \phi$ имеют тот же смысл, что и выше, опять ограничимся рассмотрением случая $\phi \in[0,1 / 2]$. Положим $\gamma_{ \pm}(u)=\widetilde{\varphi}(u) \widetilde{\varphi}(u \pm 1)$, тогда

$$
\int_{0}^{1}\left|\omega_{ \pm 1}(t, x)\right| d t=\int_{0}^{1}\left|\sum_{\ell \in \mathbb{Z}} \gamma_{ \pm}\left(2^{-j} \ell\right) \overline{X_{\ell}(\cos \pi \theta)} X_{\ell \pm 2^{j}}(\cos \pi \phi)\right| d \cos \pi \theta
$$

Разобьем этот интеграл по схеме

$$
\int_{0}^{1} d \cos \pi \theta=\int_{0}^{1} d g_{1}(\theta)+\int_{0}^{1} d g_{2}(\theta)=i_{1}+i_{2}
$$

При $\phi \neq 0$ применим $(22)$ к $X_{\ell \pm 2^{j}}(\cos \pi \phi)$. Используя неравенство (19) и учитьвая структуру носителя функции $\gamma_{ \pm}$при оценке остатка, имеем

$$
\begin{aligned}
i_{2} \leqslant & C 2^{j / 2} \int_{0}^{\phi} \frac{d \tau}{\sqrt{\cos \pi \tau-\cos \pi \phi}} \\
& \times \int_{0}^{1}\left\{\left|\sum_{\ell \in \mathbb{Z}} \gamma_{ \pm}\left(2^{-j} \ell\right) \xi_{5}\left(2^{-j} \ell \pm 1\right)\left(2^{-j} \ell\right)^{1 / 2} e^{2 \pi i \ell \tau} X_{\ell}(\cos \pi \theta)\right|\right. \\
& +\left|\sum_{\ell \in \mathbb{Z}} \gamma_{ \pm}\left(2^{-j} \ell\right) \xi_{6}\left(2^{-j} \ell \pm 1\right)\left(2^{-j} \ell\right)^{1 / 2} e^{-2 \pi i \ell \tau} X_{\ell}(\cos \pi \theta)\right| \\
& +\left|\sum_{\ell \in \mathbb{Z}} \gamma_{ \pm}\left(2^{-j} \ell\right) \xi_{7}\left(2^{-j} \ell \pm 1\right)\left(2^{-j} \ell\right)^{-1 / 2} e^{2 \pi i \ell \tau} X_{\ell}(\cos \pi \theta)\right| \\
& \left.+\left|\sum_{\ell \in \mathbb{Z}} \gamma_{ \pm}\left(2^{-j} \ell\right) \xi_{8}\left(2^{-j} \ell \pm 1\right)\left(2^{-j} \ell\right)^{-1 / 2} e^{-2 \pi i \ell \tau} X_{\ell}(\cos \pi \theta)\right|\right\} d g_{2}(\theta)+O(1)
\end{aligned}
$$

Нетрудно убедиться в том, что функция $\gamma(u)=\gamma_{ \pm}(u) \xi_{\nu}(u \pm 1)(u)^{\alpha}$ удовлетворяет условиям леммы 8 . K внутреннему интегралу применим (30) и произведем в каждом слагаемом правой части (30) замену переменной $\theta^{\prime}=\tau \pm \theta$. Учитывая, что при $\tau \leqslant \phi$ выполнено (46), на основании леммы 7 получаем $i_{2}=O(1)$. При $\phi=0$ это соотношение доказывается так же с использованием равенства (47) вместо (22). Аналогично, воспользуемся (22) для оценки $i_{1}$ при $\phi \in\left(0,2^{-j}\right)$. Применив затем (29) и (26), получим $i_{1}=O(1)$. Наконец, чтобы доказать ограниченность $i_{1}$ при $\phi \in\left(2^{-j}, 1 / 2\right)$, применим к $X_{\ell \pm 2^{j}}(\cos \pi \phi)$ соотношение (23). Используя неравенство (19) и учитывая структуру носителя функции $\gamma_{ \pm}$при оценке остаточных членов, имеем

$$
\begin{aligned}
i_{1} \leqslant & C \frac{1}{\sqrt{\phi}} \int_{0}^{1}\left\{\left|\sum_{\ell \in \mathbb{Z}} \gamma_{ \pm}\left(2^{-j} \ell\right) \xi_{1}\left(2^{-j} \ell \pm 1\right) e^{2 \pi i \ell \phi} X_{\ell}(\cos \pi \theta)\right|\right. \\
& \left.+\left|\sum_{\ell \in \mathbb{Z}} \gamma_{ \pm}\left(2^{-j} \ell\right) \xi_{2}\left(2^{-j} \ell \pm 1\right) e^{-2 \pi i \ell \phi} X_{\ell}(\cos \pi \theta)\right|\right\} d g_{1}(\theta) \\
& +O\left(\frac{2^{-j / 2}}{\sqrt{\phi}}\right) \\
+ & C \frac{2^{-j / 2}}{\sqrt{\phi}} \int_{\phi}^{1 / 2} \frac{d t}{t^{3 / 2}} \int_{0}^{t} \frac{d \tau}{\sqrt{\cos \pi \tau-\cos \pi t}}
\end{aligned}
$$




$$
\begin{aligned}
& \times \int_{0}^{1}\left\{\left|\sum_{\ell \in \mathbb{Z}} \gamma_{ \pm}\left(2^{-j} \ell\right) \xi_{9}\left(2^{-j} \ell \pm 1\right)\left(2^{-j} \ell\right)^{-1 / 2} e^{2 \pi i \ell(\tau+t-\phi)} X_{\ell}(\cos \pi \theta)\right|\right. \\
& +\left|\sum_{\ell \in \mathbb{Z}} \gamma_{ \pm}\left(2^{-j} \ell\right) \xi_{10}\left(2^{-j} \ell \pm 1\right)\left(2^{-j} \ell\right)^{-1 / 2} e^{-2 \pi i \ell(\tau+t-\phi)} X_{\ell}(\cos \pi \theta)\right| \\
& +\left|\sum_{\ell \in \mathbb{Z}} \gamma_{ \pm}\left(2^{-j} \ell\right) \xi_{11}\left(2^{-j} \ell \pm 1\right)\left(2^{-j} \ell\right)^{-3 / 2} e^{2 \pi i \ell(\tau+t-\phi)} X_{\ell}(\cos \pi \theta)\right| \\
& \left.+\left|\sum_{\ell \in \mathbb{Z}} \gamma_{ \pm}\left(2^{-j} \ell\right) \xi_{12}\left(2^{-j} \ell \pm 1\right)\left(2^{-j} \ell\right)^{-3 / 2} e^{-2 \pi i \ell(\tau+t-\phi)} X_{\ell}(\cos \pi \theta)\right|\right\} d g_{1}(\theta) \\
& +O\left(\frac{2^{-j}}{\phi}\right)
\end{aligned}
$$

Осталось применить к внутренним интегралам (29) и (26).

\section{Список литературы}

1. Ульянов П. Л. О некоторых решенных и нерешенных проблемах теории ортогональных рядов // Труды IV Всесоюзного математического съезда. Т. 2. Л.: Изд-во АН СССР, 1964. C. 694-704.

2. Ульянов П. Л. О некоторых результатах и задачах из теории базисов // Записки научн. сем. ЛОМИ. 1989. Т. 170. С. 274-284.

3. Faber G. Über dieinterpolatorische Darstelung stetiger Functionen // Jahresber. Deutsch. Math.-Verein. 1914. V. 23. P. 192-210.

4. Привалов Ал. А. О росте степеней полиномиальных базисов // Матем. заметки. 1990. T. 48. № 4. C. $69-78$.

5. Привалов Ал. А. О росте степеней полиномиальных базисов и приближении тригонометрических проекторов // Матем. заметки. 1987. Т. 42. № 2. С. 207-214.

6. Offin D., Oskolkov K. A note on orthonormal polynomial bases and wavelets // Constr. Approx. 1993. V. 9. №1. P. 319-325.

7. Lorentz R. A., Sahakian A. A. Orthogonal trigonometric Shauder bases of optimal degree for $C(0,2 \pi) / /$ J. Fourier Anal. Appl. 1994. V. 1. № 1. P. 103-112.

8. Kilgore T., Prestin J., Selig K. Orthogonal algebraic polynomial Shauder bases of optimal degree // J. Fourier Anal. Appl. 1996. V. 2. №6. P. 597-610.

9. Skopina M. Multiresolution analysis of periodic functions // East J. Approx. 1997. V. 3. № 2. P. 203-224.

10. Daubechies I. Ten lectures on wavelets // CBMS-NSF Regional Conf. Ser. in Appl. Math. V. 61. Philadelphia, PA: SIAM, 1992.

11. Сегё Г. Ортогональные многочлены. М.: Физматгиз, 1962.

12. Skopina $M$. Local convergence of Fourier series with respect to periodized wavelets // J. Approx. Theory. 1998. V. 94. № 2. P. 191-202.

13. Chanillo S., Muckenhoupt B. Weak type estimates for Cesero sums of Jaconi Polynomial series // Mem. Amer. Math. Soc. 1993. V. 102. № 487. P. 1-90.

Санкт-Петербургский государственньй университет

Поступила в редакцию

E-mail: skopina@sk.usr.lgu.spb.su 15.02.1999 и 28.12 .2000 